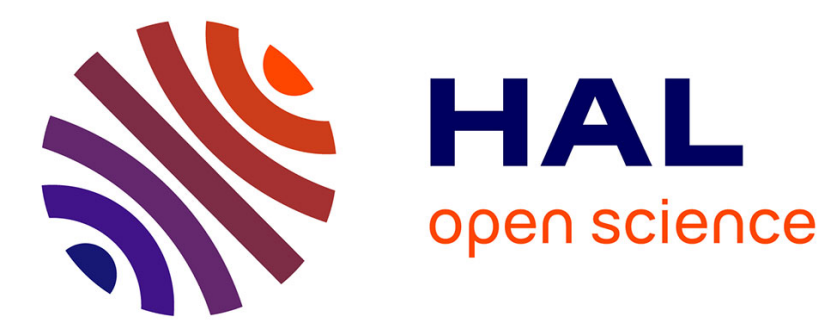

\title{
Mise en scène de la parole magistrale dans un cours de philosophie
}

\author{
Dominique Renauld
}

\section{To cite this version:}

Dominique Renauld. Mise en scène de la parole magistrale dans un cours de philosophie. Cliopsy, 2019. hal-02144539

\author{
HAL Id: hal-02144539 \\ https://hal.science/hal-02144539
}

Submitted on 6 Jun 2019

HAL is a multi-disciplinary open access archive for the deposit and dissemination of scientific research documents, whether they are published or not. The documents may come from teaching and research institutions in France or abroad, or from public or private research centers.
L'archive ouverte pluridisciplinaire HAL, est destinée au dépôt et à la diffusion de documents scientifiques de niveau recherche, publiés ou non, émanant des établissements d'enseignement et de recherche français ou étrangers, des laboratoires publics ou privés. 


\title{
Mise en scène de la parole magistrale dans un cours de philosophie
}

\author{
Dominique Renauld
}

Cet article fait suite à une thèse soutenue en novembre 2018. Intitulée Enseigner la philosophie au lycée. Hypothèses cliniques d'orientation psychanalytique, cette recherche porte sur l'enseignement de la philosophie en France, tel qu'il est actuellement dispensé dans les classes terminales des séries générales et technologiques des lycées. Elle est plus particulièrement consacrée à la manière dont des professeurs de philosophie vivent l'acte d'enseigner leur discipline et elle a été construite sur l'analyse d'entretiens cliniques non-directifs réalisés auprès de six professeurs de philosophie de l'enseignement secondaire à partir de la consigne suivante : « J'aimerais que vous me disiez, comme ça vous vient, comment vous vivez le fait d'enseigner la philosophie ».

Au cours de cette recherche, enseignant moi-même la philosophie au lycée, j'ai été particulièrement attentif aux ressentis dont font part des enseignants de cette discipline lorsqu'ils évoquent leur rapport au déploiement de la parole magistrale dans l'espace de la classe. Comment vivent-ils leur enseignement si celui-ci est, pour eux, étroitement lié à l'exercice d'une certaine démonstrativité déclamatoire et se heurte ordinairement à la difficulté de composer avec un groupe-classe dont l'attention peut être inégalement soutenue ? À quels processus psychiques inconscients cette composante irréductible de l'acte d'enseigner soumet-elle la parole magistrale lorsque sont interrogées, dans le cadre d'entretiens cliniques, des déclinaisons individuelles et singulières ?

Après avoir précisé le cadre théorique de cette recherche, je présenterai l'un des entretiens que j'ai réalisés. Puis, à partir d'une analyse du contenu latent de certains éléments d'énonciation de cet entretien, j'exposerai quelques-unes des hypothèses auxquelles celle-ci m'a mené.

\section{Cadre épistémologique de cette recherche}

Ma recherche s'inscrit dans le cadre d'une clinique d'orientation psychanalytique de recherche en sciences de l'éducation. Elle porte, en 
particulier, sur le rapport au savoir (Beillerot, Blanchard-Laville et Mosconi, 1996) de professeurs de philosophie de I'enseignement secondaire et sur des modalités psychiques de leur acte professionnel. Les notions de clinique et de rapport au savoir étant tout à fait centrales dans ma recherche, je vais en préciser certains aspects théoriques.

\section{Sur le sens du mot clinique}

Dans une synthèse qu'elle avait consacrée, en 1999, aux « sources historiques de la clinique d'orientation psychanalytique », Claudine Blanchard-Laville rappelait l'étymologie de I'adjectif clinique: du grec classique klinikê, lequel se dit « de ce qui se fait près du lit des malades, sur le patient même, et non dans les livres et par la théorie » (BlanchardLaville, 1999, p. 11), par extension, le substantif clinique désigne spécifiquement le savoir issu d'une observation directe effectuée au chevet d'un malade. Il s'agit donc d'une notion dont la formation historique s'inscrit dans une pratique de la médecine attentive aux formes diversifiées que peut recouvrer l'individuel au plan biologique. Dans le champ des sciences de l'éducation, son emploi témoigne, plus particulièrement, d'un intérêt épistémologique pour des processus et des phénomènes singuliers dont l'exemplarité, comme l'avait proposé Georges Canguilhem (1962/1994), peut être la source d'hypothèses heuristiques. Dans ce champ, lorsque l'adjectif clinique est référé à une recherche d'orientation psychanalytique, il ne recouvre ni l'acception d'une visée diagnostique (Castarède, 1983) ni le sens d'un but thérapeutique. Il désigne une démarche de production de connaissances en sciences humaines dont l'objet réside principalement dans une compréhension du fonctionnement psychique d'un sujet (Revault d'Allonnes, 1989) ou de groupes en situation (Charrier, 2009 ; Rinaudo, 2015) et dont l'un des objectifs consiste à repérer et à analyser des processus psychiques inconscients chez des protagonistes de situations d'enseignement, d'éducation et de formation (Blanchard-Laville, Chaussecourte, Hatchuel et Pechberty, 2005).

\section{La notion de rapport au savoir}

En mentionnant la notion de rapport au savoir qui, dans le champ des sciences de l'éducation, a donné lieu, depuis 1989, à de très nombreuses publications (Beillerot et al., 1996 ; Beillerot, Bouillet, Blanchard-Laville et Mosconi, 1989 ; Mosconi, Beillerot et Blanchard-Laville, 2000), je me réfère essentiellement à une hypothèse qui a été soutenue par $\mathrm{C}$. Blanchard-Laville (2010) selon laquelle, dans l'exercice de ses fonctions, un enseignant, toutes disciplines confondues, ne transmettrait pas seulement un savoir constitué et objectivé sur le plan didactique - savoir qui serait indistinct et impersonnel d'un professionnel à l'autre -, mais exposerait publiquement, dans l'espace d'enseignement, certaines modalités psychiques inconscientes selon lesquelles il se relie lui-même subjectivement au savoir qu'il enseigne. Dans cette hypothèse qui fait droit à l'idée selon laquelle, pour employer les mots de Jacky Beillerot, «l'on "est" son rapport au savoir » (Beillerot, 2000 , p. 49), un enseignant transmettrait ainsi à ses élèves, le plus souvent 
à son insu, ses «propres modalités de rapport au savoir » davantage que « des objets de savoir bien circonscrits et objectivables » (Blanchard-Laville, 2013a). Selon I'analyse qu'en propose C. Blanchard-Laville, le rapport au savoir désignerait en ce sens tant la « partie vivante » d'un enseignant que « ses traumatismes, ses impensés, ses enkystements » (Blanchard-Laville, 2013b, p. 127).

\section{Clinique et réalité psychique inconsciente}

À ce titre, l'hypothèse de l'existence d'un inconscient psychique dynamique, telle qu'elle fut élaborée par Freud1, constitue une référence théorique centrale de ma recherche. Cette prise en considération de l'inconscient implique pour moi que, comme le rappelait Freud en ouverture de son ouvrage théorique de 1923, Le moi et le ça, le conscient ne serait pas la qualité essentielle du psychique, mais que celui-ci serait également déterminé par un ensemble de processus et de mécanismes inconscients justiciables d'une causalité psychique qui échapperait en grande partie à la psyché. Il s'agit ainsi, comme l'avait proposé Freud - et comme le soulignaient les philosophes Renée Bouveresse-Quilliot et Roland Quilliot dans leur ouvrage Les critiques de la psychanalyse -, de faire droit à I'hypothèse de «l'émergence d'un niveau de réalité nouveau, le vécu subjectif, impossible à décrire dans les termes des sciences exactes» (Bouveresse-Quilliot et Quilliot, 1991, p. 100).

Dans cette perspective, ma démarche, en tant que chercheur clinicien, consiste à tenter de repérer certains processus et mécanismes inconscients tels que des conflits psychiques internes qui pourraient sous-tendre le discours d'enseignants sollicités afin d'évoquer leur acte professionnel dans le cadre d'entretiens cliniques. Je fais en effet I'hypothèse - suivant en cela la proposition de C. Blanchard-Laville selon laquelle des processus inconscients «agissent à l'insu même des protagonistes » (BlanchardLaville, 1999, p. 18) de situations professionnelles et « ne sont pas lisibles ni pour le profane ni pour les sujets eux-mêmes» (Ibid.) - que certains éléments linguistiques et paralinguistiques pourraient signer quelque chose de l'ordre de fantasmes inconscients (Kaës, 1975) sous-jacents aux discours que tiennent ces professionnels sur leurs pratiques d'enseignement.

\section{Le chercheur et son appareil psychique}

$\mathrm{Ce}$ modèle théorique requiert une instrumentation congruente avec l'épistémologie de la recherche clinique d'orientation psychanalytique : qu'il s'agisse d'un entretien clinique (Castarède, 1983 ; Yelnik, 2005, 2007) ou d'une observation (Chaussecourte, 2010a, 2010b), c'est en effet son propre appareil psychique qu'un chercheur clinicien sollicite afin de repérer, d'identifier et d'analyser des phénomènes et des processus psychiques inconscients. De ce fait, et dans la mesure où un chercheur clinicien est également partie prenante de la relation qu'il entretient avec son objet d'étude, il est aussi un élément constitutif et irréductible de sa propre recherche (Barus-Michel, 1986 ; Mosconi, 1986). Cette modalité spécifique
1. Notamment dans L'inconscient (2013), non publié du vivant de Freud, rédigé en 1915. 
de l'instrumentation clinique requiert donc que, consubstantiellement à la recherche qu'il effectue sur son objet, il puisse accomplir un travail psychique personnel, c'est-à-dire qu'il analyse sa propre implication dans son objet d'étude (Devereux, 2012), certains enjeux personnels et professionnels ainsi que des ressentis ou des investissements psychiques contre-transférentiels (Berdot, Blanchard-Laville et Camara Dos Santos, 1997) dans la relation avec les sujets de sa recherche. Ce travail d'élaboration psychique (Blanchard-Laville, 2008, p. 97) doit permettre au clinicien de mieux appréhender son «contre-transfert-de-chercheur » (Chaussecourte, 2017) tout au long du processus de recherche (BlanchardLaville et Geffard, 2009). Cette précaution méthodologique n'a pas seulement vocation à éliminer le plus d'interférences possible dans le recueil de données empiriques en disposant le chercheur à prendre plus clairement conscience d'attentes personnelles qui risqueraient d'orienter ses hypothèses interprétatives dans le sens d'une subjectivité qui, faute d'être élaborée (Blanchard-Laville, 1999), serait alors « occultée » (Cifali, 2008) ou, du moins, insuffisamment «travaillée » (Cifali, 1999). Elle doit aussi faciliter une analyse du matériel recueilli. Dans cette approche, en effet, l'appareil psychique du chercheur clinicien est un outil de connaissance à part entière (Bertrand, 2004, p. 1647).

Mon cadre théorique de recherche ayant été précisé, je vais à présent expliciter certains aspects de l'un des entretiens que j'ai réalisés.

\section{Présentation de l'entretien}

Sur les six entretiens cliniques que j'ai enregistrés au cours de ma recherche, celui dont je rapporte ici certaines analyses a été réalisé en 2014 dans le lycée d'exercice d'une enseignante à laquelle j'ai donné le pseudonyme de Jocelyne.

\section{Portrait de Jocelyne et circonstances de l'entretien}

Jocelyne est une enseignante d'une cinquantaine d'années qui, comme elle me l'expliquera au cours de l'entretien, enseigne la philosophie au lycée depuis vingt-sept ans. Dès notre rencontre, je me surprends à relever un détail physique qui retiendra plus particulièrement mon attention lors de l'analyse ultérieure du contenu latent de son discours : sa voix ainsi que les traits de son visage, assez durs, presque anguleux, m'évoquent ceux d'un homme et, plus particulièrement, ceux d'un acteur comique français des années 80. À son arrivée dans le hall de son établissement, elle propose que nous nous installions dans une pièce située dans la partie administrative, à l'écart des lieux habituellement fréquentés par les lycéens. En entrant dans cette pièce, je constate que les rideaux ont été tirés ; elle est donc plongée dans l'obscurité et la seule lumière présente est projetée par des néons situés au plafond. De ce fait, cet endroit produit sur moi des effets de clairobscur que j'associe à certains tableaux de Georges de La Tour et, en particulier, au tableau intitulé Le nouveau né : sombre à l'arrière-plan, 
éclairée en son centre, là où nous nous sommes installés pour l'entretien, la pièce crée en moi une impression d'enveloppement.

À I'issue de notre rencontre, j'associe le choix de ce lieu à certains éléments du discours de Jocelyne au cours de l'entretien. En effet, elle a choisi une pièce dont l'éclairage artificiel m'évoque aussi les jeux d'ombres et de lumières utilisés au théâtre, éléments constitutifs d'une mise en scène dont elle m'explique la fonction prépondérante dans sa propre pratique d'enseignement. Je me demande donc ce que Jocelyne, inconsciemment, pourrait à la fois chercher à occulter et à dévoiler d'elle-même en privilégiant une lumière qui, d'un côté, dissimule certains aspects de la pièce et, de l'autre, enveloppe nos corps d'un halo.

Cet entretien, depuis l'instant où j'énonce ma consigne jusqu'au moment où j'en arrête l'enregistrement, a duré exactement trente minutes. Les analyses que j'en propose comportent trois moments : dans un premier temps, je rapporterai un résumé du contenu manifeste chronologique de I'entretien, puis, partant d'une analyse de certains aspects du contenu latent de ce dernier, j'exposerai plusieurs hypothèses relatives au rapport de Jocelyne à l'acte d'enseigner la philosophie.

Les citations issues de l'entretien ont été mises en italiques. Les barres obliques $(/ /)$ renvoient à des silences dont la durée peut varier de une à quelques secondes et les didascalies ont été placées entre parenthèses.

\section{Résumé chronologique du contenu manifeste}

Jocelyne débute l'entretien en m'exposant son attrait pour l'enseignement de la philosophie. Elle évoque ainsi son «goût pour la transmission » et le fait qu'« avant même de connaître l'existence de la philosophie », elle aimait déjà «transmettre » à d'autres personnes ce qu'elle avait appris. Elle m'explique également avoir choisi d'enseigner la philosophie parce qu'il ne lui semblait pas possible d'enseigner une autre discipline et en raison du très grand intérêt qu'elle trouvait à cette matière d'enseignement et aux savoirs que celle-ci mobilise. Elle considère ainsi la philosophie, non seulement comme «la plus importante» de toutes les disciplines, mais également comme celle «qui va sauver le monde ». Très tôt dans l'entretien, dès la deuxième minute, Jocelyne reprend ensuite les termes de ma consigne - «comment je vis » - en expliquant qu'elle transmet son enseignement en recourant aux ressources du spectacle et de la mise en scène : elle dit en effet avoir «l'impression de jouer tous les rôles», de jouer «les philosophes », de jouer des dialogues et d'imiter des voix «comme dans les dessins animés ». Puis, enchaînant une série de propositions consacrées au plaisir qu'elle éprouve à exercer son métier, elle me fait part de cette « grande satisfaction » qu'elle ressent et me dit : «je suis payée pour faire ce que j'ai envie de faire ». Ce plaisir lui semble lié au fait qu'on lui «donne un public » pour l'écouter «parler de philosophie ». Cette situation est pour elle inhabituelle. Elle remarque en effet qu'elle n'a pas l'occasion, en dehors des cours qu'elle donne au lycée, d'être écoutée de cette manière. Elle m'explique à la fois qu'elle ne se limite pas à transmettre des éléments de 
savoir constitués en vue de l'examen final, mais qu'elle cherche à dépasser le cadre scolaire de l'enseignement en transmettant également « une sorte de modèle de vie » et de questionnement. Elle m'explique ainsi qu'elle tente également, de cette manière, de toucher «I'humanité de l'élève » en faisant entrevoir à celui-ci « ce que pourrait être l'humanité ».

Elle aborde ensuite la question de la « souffrance au travail » en affirmant qu'elle n'en a pas du tout. Elle déclare en effet être «contente de venir travailler » même si elle concède qu'elle est « aussi contente » quand elle a fini. Elle m'explique en effet qu'elle dépense «beaucoup d'énergie » dans la mise en scène qu'elle vient d'évoquer et que, de ce fait, il ne lui est pas possible d'enseigner plus de «trois ou quatre heures» par jour. Elle réinterroge alors la thématique de la théâtralité et explique qu'elle «impose le spectacle » et qu'elle ne donne peut-être pas suffisamment la parole à ses élèves. Elle dit en effet qu'elle ressent de l'ennui lorsque les élèves prennent la parole en raison des répétitions que comporte leur discours.

Après qu'elle a murmuré qu'elle n'allait pas pouvoir parler une demi-heure, je formule une relance: "aller vers les élèves vous avez dit tout à I'heure ». Jocelyne explique alors qu'elle a avec ses élèves une «assez bonne relation » et ne leur donne jamais de punitions. Elle s'efforce en effet de «jouer sur le dialogue [...] sur la relation de confiance ». Cette attitude qu'elle adopte à l'égard des élèves lui fait dire qu'elle confond peut-être «enseignement et éducation ». Elle justifie cette attitude en évoquant un aspect de son enseignement qu'elle a déjà abordé, à savoir le fait que la philosophie «fait de nous des hommes » et qu'elle comporte donc une part $\mathrm{d}^{\prime}$ « exemplarité dans la conduite ». Elle explique en ce sens qu'elle s'efforce elle-même de conformer ses actes à son discours. Elle dit avoir le sentiment que ses élèves perçoivent cette concordance, laquelle témoigne, selon elle, d'une «certaine forme de sagesse ». Elle souhaite en effet donner de la philosophie non pas simplement l'image d'une «transmission de savoir » ou de méthodes, mais celle de «quelque chose de proprement humain ».

Elle marque alors une courte pause et je formule une nouvelle relance : « et vous avez parlé de mise en scène tout à l'heure ». Elle précise le sens qu'elle donne à cette notion de mise en scène. Elle explique qu'elle tente $\mathrm{d}^{\prime}$ « incarner» les idées philosophiques dans son corps. Selon elle, le discours philosophique est pour les élèves « excessivement conceptuel ». Elle évoque ainsi les personnages au moyen desquels elle met en scène ces idées. Elle prend l'exemple d'Astérix qu'elle relie à la dialectique du maître et de l'esclave de Hegel. Ce développement donne lieu à l'expression d'un ressenti au sujet du rapport des élèves à ce qu'elle appelle le «conceptuel ». Elle explique en effet avoir le sentiment que les élèves ont peu d'intérêt pour les « combats d'idées »; ils paraissent plus intéressés par des considérations pratiques telles qu'«avoir un travail» que par des considérations intellectuelles ou politiques telles que «se battre pour la liberté ». Elle poursuit en soutenant que, pour elle, l'humanité ne peut pas «se retrouver dans le fait d'avoir une famille une maison un chien et voire 
peut-être une piscine ». C'est en ce sens qu'elle entend le fait de donner à ses cours « une autre dimension que l'enseignement ».

Elle explique qu'elle a le sentiment, en tenant ce discours, de ne pas s'en tenir à la mission que l'Éducation nationale lui a confiée, laquelle consiste, selon elle, à apprendre aux élèves à savoir faire une dissertation et une explication de texte. Elle occuperait ainsi une place que d'autres ne tiendraient pas et serait, selon son expression, «la cerise sur le gâteau ». Jocelyne conclut l'entretien en disant «je sais plus quoi dire », phrase sur laquelle j'arrête en disant «ok».

\section{Analyse de l'entretien}

\section{De l'enseignement comme d'un spectacle}

À la fin de la deuxième minute de l'entretien, Jocelyne associe le geste de transmettre à l'idée d'un «spectacle » et d'une «mise en scène ». À cette occasion, m'expliquant qu'elle est «très très tournée vers la parole », elle me confie avoir «l'impression de jouer tous les rôles » et me dit : « je joue les philosophes je joue les dialogues je me mets en scène en gestes ». Explicitant son ressenti sur ce point, Jocelyne enchaîne alors deux propositions dont la succession me surprend. Concédant ne pas savoir «quel est l'effet pédagogique » d'une telle mise en spectacle de son enseignement, elle me dit aussitôt qu'« après euh / c'est une satisfaction à travailler » et me confie ce qu'elle affirme dire «toujours » à ses élèves: «je suis payée pour faire ce que j’ai envie de faire ». Puis, précisant ce dernier point, elle ajoute immédiatement : «on me donne un public pour m'écouter parler de philosophie alors que souvent dans la vie courante je ne vais pas nécessairement avoir l'occasion d'être face à des gens qui ont envie d'écouter un certain discours ». En effet, soutient-elle, «là on a fait asseoir devant moi des gens qui ne sont pas toujours totalement libres de leur présence mais euh qui du moins sont là pour m'écouter ». Enfin, conclutelle, dans la mesure où l'enseignement lui permet de parler de ce dont elle a envie «face à un public qui n'est pas tout à fait conquis et qui reste à conquérir mais qui du moins est déjà assez là », elle me dit éprouver « une assez grande satisfaction ».

Comme elle semble le concéder elle-même en affirmant ne pas savoir si, en se mettant «beaucoup en scène », elle fait «comme on doit faire » et comme m'incline aussi à le supposer le rire qu'elle a aussitôt, Jocelyne, selon toute vraisemblance, a conscience de ce que ce rapprochement en classe, entre enseignement de la philosophie et spectacle, peut avoir de surprenant et d'inattendu sur le plan pédagogique. De mon côté, lorsque, par identification à la part psychique du moi professionnel de Jocelyne, je tente d'élaborer les résonances que suscite en moi l'évocation de cette théâtralisation de sa pratique d'enseignement, je ressens un certain embarras: dans mon ressenti contre-transférentiel de professeur de philosophie, je ne parviens pas sans peine à concevoir la logique d'une telle 
mise en scène. Je me demande, en ce sens, si, en émettant ce rire, elle ne s'épargnerait pas une explicitation de cette évocation pédagogique qui pourrait être plus coûteuse pour elle sur le plan psychique. Dans son ouvrage de 1905 consacré à l'humour, Le mot d'esprit et sa relation à l'inconscient, Freud analyse le processus du rire dans la formation des traits d'esprit comme l'expression d'une «économie de dépense » psychique (Freud, 1905/1988, p. 332) et fait I'hypothèse qu'en riant nous nous épargnons de longues et fastidieuses explications.

Par ailleurs, dans ce contexte d'évocation de sa pratique d'enseignement, le pronom réfléchi me, auquel elle a recours dans la phrase «je me mets en scène », retient mon attention. En effet, Jocelyne ne dit pas seulement qu'elle illustre son discours par le biais d'une théâtralisation dont la vocation serait, à ses yeux, pédagogique. Elle affirme qu'elle se met elle-même en scène. Très tôt dans l'entretien, le dispositif du cours tel qu'elle le décrit m'évoque fortement la scène d'un théâtre sur laquelle elle tiendrait un rôle central. Cette association du spectacle et de l'enseignement n'allant de soi ni pour moi ni pour Jocelyne elle-même - elle dit ne pas savoir «quel est l'effet pédagogique » d'une telle mise en scène -, j'ai cherché à comprendre le sens psychique inconscient que pourrait recouvrer sa manière d'éviter toute explicitation.

\section{Des élèves-spectateurs}

Le fait de ne pas être en mesure d'évaluer les effets pédagogiques d'une telle mise en scène sur ses élèves semble avoir assez peu d'importance à ses yeux. La satisfaction qu'elle dit ressentir à se mettre ainsi en scène - et non le plaisir ou l'intérêt de ses élèves - paraît en effet l'emporter sur toute autre considération, comme si le fait qu'elle dise être «payée pour faire »ce qu'elle a envie de faire justifiait à ses yeux une telle mise en spectacle.

Les mots qu'elle emploie pour évoquer ses élèves et les modalités de sa relation à ceux-ci - un «public » qui serait là pour l'« écouter parler de philosophie »- me confortent dans cette impression. Jocelyne tient un discours dont certains traits m'évoquent ce que Claude Pujade-Renaud, décrivant le professeur tout à la fois comme un acteur et un metteur en scène, appelle une «centration narcissique» (Pujade-Renaud, 2005, p. 140). Ainsi Jocelyne dit : «on me donne un public » mais, alors que le public d'un spectacle vient librement, elle décrit les élèves comme des sujets qui seraient contraints de l'écouter: «là on a fait asseoir devant moi des gens qui ne sont pas toujours totalement libres de leur présence mais euh qui du moins sont là pour m'écouter ». Si Jocelyne concède qu'elle ne «donne pas assez la parole» à ses élèves, en tant que professeur de philosophie, moi-même, je suis surpris par la justification qu'elle apporte, tant elle me semble dénuée de toute préoccupation pédagogique : d'une part, dit-elle, «peut-être que je m'ennuie quand ils parlent »; d'autre part, ce que lui disent ses élèves, «c'est quelquefois tellement répétitif [...] tellement attendu », qu'« au bout d'un moment j'avoue j'en ai relativement assez de les entendre ». C'est la raison pour laquelle Jocelyne explique 
qu'« avant même de laisser la parole aux élèves », elle a «tendance à dire bon voilà ce que vous allez me dire et faire une sorte de liste de ce qu'ils vont dire voilà pourquoi il ne faut pas le dire et mais avant de leur avoir laissé dire ». Puis, elle ajoute : "là encore c'est moi qui impose le spectacle qui tire les ficelles », ce qui évoque la « concentration des fonctions » dont parle C. Pujade-Renaud qui confère à l'enseignant acteur et metteur en scène « un pouvoir quasi totalitaire » sur ses élèves (Ibid.). Comme ces différents éléments d'énonciation m'inclinent à en faire I'hypothèse, cette mise en scène de sa parole ne relève pas seulement d'un projet pédagogique conscient, mais semble venir au service de ses attentes narcissiques. Ainsi que je vais en effet l'établir, certains mouvements psychiques inconscients singuliers, inhérents à cette manière de faire et, en ce sens, spécifiquement à l'œuvre pour Jocelyne, paraissent également sous-tendre sa pratique professionnelle.

\section{Les petites voix des personnages de dessins animés}

Ainsi, m'expliquant qu'elle «utilise peu les moyens technologiques modernes » et qu'elle est «très très tournée vers la parole », Jocelyne dit qu'elle fait «à la limite comme dans les dessins animés euh les petites voix des personnages (rire) ». À la relecture de la transcription de cette séquence de l'entretien, cette évocation d'un aspect de sa pratique professionnelle, ressaisie à travers mes ressentis contre-transférentiels d'enseignant, n'est pas sans me surprendre. En effet, l'un des objectifs pédagogiques de l'enseignement de la philosophie est de contribuer à former une posture critique chez des élèves en favorisant l'accès de ceux-ci « à l'exercice réfléchi du jugement »(BOEN, 2003). À cet égard, l'exemple donné par le maître à ces derniers constitue, au moins dans les séries générales, un élément consubstantiel de cette transmission (Engel, 1998 ; Kambouchner, 2007). Je m'interroge donc, depuis ma position de professeur de philosophie, sur la pertinence démonstrative d'une telle mise en scène à des fins pédagogiques. Que peuvent en effet ressentir des élèves qui, en écoutant leur professeur les initier à l'expérience du raisonnement philosophique, entendent celui-ci imiter les «petites voix » de personnages, comme dans les dessins animés ?

Dans une première phase d'élaboration psychique de cette séquence de l'entretien, je me suis demandé si le diminutif qu'emploie Jocelyne - «petites voix» - n'aurait pas, pour elle, inconsciemment, la valeur d'une identification à son moi d'enfant. En effet, l'enfant grandit, dans un premier temps, dans un univers où les dessins animés sont d'abord la mise en scène d'un monde peuplé d'enfants ou d'animaux aux voix d'enfants, nécessairement fluettes, petites, c'est-à-dire aiguës. On pourrait ainsi faire I'hypothèse que, dans ce jeu qui consiste à se mettre en scène au moyen de «petites voix», ce serait une part psychique de son moi d'enfant que Jocelyne exposerait inconsciemment dans l'espace de la classe. Dans un article publié en 1908 et intitulé Le créateur littéraire et la fantaisie, Freud développe I'hypothèse d'une diffraction du moi de l'écrivain à travers des 
personnages qu'il crée. En exposant des parties de lui-même - des sentiments ou des impressions, par exemple - à travers de multiples personnages, le romancier ne parlerait en fin de compte que de lui-même et trouverait ainsi, dans l'écriture, un gain psychique de plaisir narcissique que la société ne peut lui concéder en temps ordinaire. Je me demande si Jocelyne, en imitant des «petites voix », n'éprouverait pas un gain de plaisir psychique similaire. En effet, dès la première minute de l'entretien, elle fait allusion à une position psychique enfantine précoce qui $\mathrm{m}^{\prime}$ incline à supposer qu'elle a pu concourir à étayer une part de son narcissisme d'enfant: " avant même de connaître l'existence de la philosophie j'avais déjà un goût pour la transmission puisque euh j'ai toujours aimé dès que j'apprenais quelque chose le montrer le dire et le transmettre à quelqu'un d'autre ».

\section{Mettre en scène le conceptuel}

Dans un second temps d'élaboration de cette séquence, cherchant à comprendre à quelle vocation pédagogique pourrait obéir ce rapprochement entre théâtre et enseignement, j'ai d'abord supposé que Jocelyne cherchait à donner vie à des notions abstraites en illustrant celles-ci par une mise en spectacle qui, mêlant étroitement mise en scène corporelle et expression vocale, donnerait du corps à sa démonstration. En effet, à la vingt-troisième minute de l'entretien, Jocelyne m'expliquera qu'elle s'efforce $d^{\prime}$ «incarner » dans sa personne «les idées philosophiques », d'« incarner dans le corps » ou de «faire prendre chair à quelque chose » qui, pour ses élèves, est « excessivement conceptuel ». Elle chercherait, par exemple, à illustrer la dialectique du maître et de l'esclave de Hegel (1807/1941) par une "sorte de mise en scène pour expliquer euh une dialectique qui est / conceptuelle », le conceptuel n'étant «quand même pas tout à fait bien maîtrisé par les élèves ».

\section{La mise en scène du corps de l'enseignant}

Pour surprenante qu'elle puisse paraître en première approche, dans la mesure où elle s'éloigne d'une représentation académique de la parole magistrale dont de nombreux commentateurs de l'enseignement de la philosophie au lycée ont souligné la récurrence dans l'histoire de cette discipline (Pinto, 1983, 2000 ; Poucet, 1999 ; Roman, 1988), une telle mise en scène n'a rien d'extraordinaire dans l'enseignement. Dans une section de son ouvrage Le corps de l'enseignant dans la classe intitulée « La mise en scène du corps et du personnage », $\mathrm{C}$. Pujade-Renaud relève en effet, dans le discours des enseignants qu'elle a rencontrés, de nombreuses similitudes entre théâtre et enseignement. Ainsi rapporte-t-elle le témoignage d'un enseignant qui, décrivant sa pratique professionnelle, «pratique très magistrale » (Pujade-Renaud, 2005, p. 75), n'hésitait pas à affirmer : «c'était une pratique essentiellement centrée sur un seul personnage qui était moi »(Ibid.). Aussi me suis-je demandé si cette mise en spectacle de la parole de Jocelyne ne pourrait pas procéder également d'une stratégie inconsciente par laquelle, comme l'écrit C. Pujade-Renaud, plutôt que 
« d'être passivement exposé aux regards, l'enseignant opère une mise en scène active du corps » (Id., p. 75).

En effet, comme l'a proposé cet auteur, si le « champ du para-verbal, - gestes, attitudes, voix, etc. » peut apparaître « comme un adjuvant du processus de transmission du savoir » (Id., p. 77), il peut être également interprété «comme ce qui tente de masquer les lacunes du savoir, ou parfois la propre lassitude de l'enseignant à l'égard d'un savoir ressassé » (Ibid.). Sa fonction ne serait donc pas seulement pédagogique, même si, comme Jocelyne l'affirme, sa mise en scène de la dialectique du maître et de l'esclave à travers l'évocation du « combat des chefs dans Astérix » participe avant tout, consciemment du moins, d'un tel projet. On peut se demander si cette mise en spectacle de son enseignement ne viserait pas, inconsciemment, à compenser ce que C. Pujade-Renaud appelle «l'insuffisance de séduction intrinsèque de la connaissance » (Ibid.), insuffisance à laquelle «l'enseignant doit suppléer par les ressources séductrices des arts du théâtre » (Ibid.) afin de soutenir son effort pour retenir l'attention de ses élèves. Dans cette hypothèse, la théâtralité dont Jocelyne dit avoir fait un élément central de sa pratique professionnelle, pourrait constituer un « complément de son autorité » (Id., p. 78).

\section{Une mise en scène de soi ?}

Puis, à la faveur d'une relecture de la transcription de l'entretien et de nouvelles élaborations psychiques, je me suis demandé si cette valorisation de la mise en scène dans le discours de Jocelyne ne serait pas également infléchie, inconsciemment, par des préoccupations latentes qui porteraient sur certains traits physiques, traits dont j'ai souligné précédemment qu'ils m'évoquaient davantage ceux d'un homme que ceux d'une femme. Ce goût affirmé de Jocelyne pour la mise en scène renvoie à son intérêt précoce pour une certaine théâtralisation de soi étroitement liée à l'ostension d'un savoir. Dans un fragment que j'ai déjà évoqué, portant sur l'histoire de son rapport au savoir et à la transmission, souvenir non daté mais antérieur, selon ses propres dires, à sa découverte de «l'existence de la philosophie », elle me dit avoir «toujours aimé », dès qu'elle apprenait quelque chose, «le montrer le dire et le transmettre à quelqu'un d'autre ». Le repérage de cet élément biographique avec une composante significative d'exhibition, comme m'incline à le supposer l'emploi qu'elle fait du verbe montrer, m'a amené à réinterroger, au plan psychique inconscient, l'importance de cette mise en scène de soi et de cette théâtralisation du savoir.

\section{Du féminin pour faire du lien}

Deux postures distinctes se dégagent assez nettement dans le discours que tient Jocelyne. D'un côté, lorsqu'il s'agit de décrire des aspects éducatifs de son lien aux élèves, les mots qu'elle emploie pour décrire des modalités de cette relation m'évoquent certaines qualités psychiques que Georges Gaillard attribue à « un féminin de liaison » (Gaillard, 2008, 2011/2018). En effet, Jocelyne dit qu'elle «joue beaucoup sur le lien de confiance » et que, 
n'ayant «pas trop par exemple d'autoritarisme à l'égard des élèves », elle ne punit «jamais» ceux-ci - tout en modalisant quelque peu ce dernier propos («enfin ») -, «qu'en vingt ans », elle a «peut-être donné trois ou quatre punitions dans des cas extrêmes », et conclut qu'elle joue «toujours sur le dialogue sur la relation de confiance sur aller parler avec un élève à la sortie d'un cours parler dans le couloir enfin d'établir quelque chose ». Elle me donne ainsi l'impression de définir sa démarche éducative en insistant sur une « capacité de liaison psychique » (Gaillard, 2018, p. 113).

\section{Jouer I'homme}

De l'autre côté, lorsqu'elle évoque l'effort qu'elle fournit, en classe, pour mettre en adéquation son discours et sa conduite, Jocelyne insiste sur le fait qu'il s'agit pour elle de transmettre «quelque chose de proprement humain », de nature à faire de sa vie " un acte de philosophie en tant que tel » qui soit «perceptible par les élèves». Elle dit alors qu'elle vise à montrer à ceux-ci «le comment c'est qu'être homme devenir homme bien jouer l'homme ». Puis elle évoque deux philosophes, Montaigne et Sartre : « ça doit être de Montaigne ça (petit rire) bien faire l'homme il dit pas bien jouer bien faire l'homme jouer ça serait plutôt du Sartre » et conclut cette séquence en affirmant : «donc voilà je mon enseignement c'est bien faire l'homme devant les élèves euh (petit rire) pour donner un exemple de ce que ça pourrait être que de faire de la philosophie ».

Enfin, pour justifier le fait que cet enseignement ne consiste pas à transmettre seulement des connaissances, elle dit, au sujet de ses élèves, qu'elle a envie de se «battre contre eux en leur disant mais ça peut pas être que ça votre vie et il faut qu'elle ait une autre dimension », comme si, dans son ressenti, le combat pour des idées impliquait, à l'opposé du féminin de liaison que j'évoquais plus haut, une position nécessairement virile, voire masculine.

\section{Le savoir comme attribut phallique}

Dans le discours de Jocelyne, le savoir, qu'il soit ou non philosophique, apparaît ainsi sous les traits d'un avoir, d'un bien particulier dont elle serait la détentrice et dont l'exhibition contribuerait à la mettre en valeur, comme si, selon I'hypothèse de $C$. Pujade-Renaud, le savoir enseigné était « investi d'un pouvoir phallique »(Pujade-Renaud, 2005, p. 138). Plusieurs éléments d'énonciation m'amènent à faire cette hypothèse.

Outre le fait que, comme je viens de l'évoquer, Jocelyne établit un lien explicite entre «montrer» ce qu'elle sait et «le transmettre à quelqu'un d'autre », elle m'explique également qu'elle s'efforce, en classe, «de de de montrer enfin d'avoir une certaine exemplarité dans la conduite ». Par exemple, si elle explique à des élèves ce que sont la justice et l'injustice, elle veille «toujours à ne pas commettre d'injustices à l'égard d'élèves ». Dans ce cas également, il s'agit, pour elle, de montrer "qu'il y a un un lien entre le savoir et la sagesse entre la connaissance et le comportement », de montrer, par sa «manière d'être », «quelque chose de la philosophie », la 
finalité d'une telle conduite étant de «montrer» aux élèves «que la philosophie ça fait de nous des hommes ».

\section{La voix de l'enseignante}

Ces différents éléments d'énonciation, depuis l'évocation des petites voix de personnages de dessins animés jusqu'à certains traits d'identification à des positions masculines, m'ont amené à me demander si certains aspects du discours de Jocelyne ne seraient pas infléchis inconsciemment par une fantasmatique de formation que C. Pujade-Renaud appelle «l'être-femme » de l'enseignante, « cet "être-femme" placé sur la scène pédagogique » dont l'essence même se caractériserait par une « faille, un manque [...] supposés intrinsèques » (Id., p. 63). Je me suis demandé, en particulier, si l'imitation de «petites voix» ne serait pas, pour Jocelyne, un moyen inconscient d'annuler le fait qu'en général, la voix est « révélatrice d'une différenciation sexuelle » (Id., p. 68) et que la voix féminine, en particulier, « dans des registres aigus désagréables pour le locuteur comme pour les auditeurs » (Ibid.), ne serait pas à l'avantage de l'enseignante. Ainsi, je ferai I'hypothèse que l'imitation de petites voix, à la faveur de leurs résonances enfantines, serait peut-être, pour elle, un moyen inconscient d'exercer un certain contrôle sur un attribut essentiel de sa fonction d'enseignante, consubstantiel à la parole magistrale, mais qui est également un élément physique constitutif de son identité sexuée, sa voix.

\section{Faire cours et mettre en scène}

$\mathrm{Si}$, comme I'a souligné Philippe Chaussecourte (2014, p. 69), I'analyse d'un entretien clinique ne permet pas de repérer, dans le discours que tient un enseignant sur sa pratique professionnelle, ce qui pourrait être du ressort de son transfert didactique, cette «empreinte psychique» qui modèle la « rencontre didactique » (Blanchard-Laville, 2010, p. 150-151), néanmoins, de nombreux indices m'ont amené à faire I'hypothèse que Jocelyne a peutêtre saisi l'occasion de notre rencontre pour évoquer, voire pour réinterroger, en ma présence, dans l'ici et maintenant de l'entretien, certains aspects de son rapport à l'exercice de son métier, aspects qui, à ses yeux, seraient distinctifs d'une certaine théâtralisation de la parole magistrale. Ainsi, à l'issue de l'entretien, Jocelyne exprimera son souhait d'avoir, de ma part, un retour sur ses pratiques pédagogiques. Dans son discours, en effet, l'acte d'enseigner la philosophie apparaît en grande partie à travers son évocation de la mise en spectacle de ses cours. En raison de nombreuses résonances contre-transférentielles liées à mon statut de chercheur et de professeur de philosophie, Jocelyne suscite en moi l'impression de faire classe, c'est-à-dire de composer avec un groupe, voire de faire scène en cherchant à capter et à retenir l'attention de ses élèves, davantage que de faire cours, au sens de transmettre démonstrativement des savoirs constitués sur le modèle académique de la leçon magistrale. Une certaine théâtralisation de sa parole pourrait ainsi participer d'un expédient qu'elle mettrait en œuvre afin de faire face à un «public » qui, à la différence de celui des théâtres, n'est pas nécessairement très attentif. À cet égard, 
comme je me suis efforcé de le proposer, cette mise en spectacle, constitutive d'une mise en scène de soi, qui concourt manifestement à étayer Jocelyne dans son acte professionnel, pourrait aussi ressortir de ce que C. Blanchard-Laville appelle une «jouissance à être vu» (2013b, p. 153). Cette jouissance pourrait être en lien avec une certaine souffrance psychique que Jocelyne semble ressentir lorsqu'elle évoque la dépense d'énergie qu'elle fournit pour capter l'attention de ses élèves.

\section{Conclusion}

Dans l'enseignement de la philosophie, comme dans d'autres disciplines, le déploiement de la parole magistrale d'un-e enseignant-e est infléchi par des processus psychiques inconscients et par des modalités psychiques propres de lien au savoir enseigné. Le repérage de certains de ces processus dans le discours de Jocelyne conforte I'hypothèse de C. Blanchard-Laville selon laquelle les enseignants «transmettent bien plus leurs propres modalités de rapport au savoir ou aux savoirs que des objets de savoir bien circonscrits et objectivables »(2013a, p. 93-94). Ainsi, cette étude du discours d'un professeur de philosophie met en évidence la dimension psychique inconsciente qui sous-tend le thème de la théâtralisation de la parole magistrale, du moins dans le discours sur sa pratique professionnelle, sinon dans sa pratique elle-même.

\section{Références bibliographiques}

Barus-Michel, J. (1986). Le chercheur, premier objet de la recherche. Bulletin de psychologie, 377(XXXIX), 801-804.

Beillerot, J. (2000). Le rapport au savoir. Dans N. Mosconi, J. Beillerot et C. BlanchardLaville (dir.), Formes et formations du rapport au savoir (p. 39-57). Paris : L'Harmattan.

Beillerot, J., Blanchard-Laville, C. et Mosconi, N. (dir) (1996). Pour une clinique du rapport au savoir. Paris : L'Harmattan.

Beillerot, J., Bouillet, A., Blanchard-Laville, C. et Mosconi, N. (1989). Savoir et rapport au savoir. Élaborations théoriques et cliniques. Paris : Éditions universitaires.

Berdot, P., Blanchard-Laville, C. et Camara Dos Santos, M. (1997). La construction de l'espace psychique dans la classe. Dans C. Blanchard-Laville (dir.), Variations sur une leçon de mathématiques. Analyse d'une séquence : « L'écriture des grands nombres » (p. 217-258). Paris : L'Harmattan.

Bertrand, M. (2004). Questions épistémologiques. Revue française de psychanalyse, 68(5), 1645-1650. doi: 10.3917/rfp.685.1645

Blanchard-Laville, C. (1999). L'approche clinique d'inspiration psychanalytique : enjeux théoriques et méthodologiques. Revue française de pédagogie, 127, 9-22.

Blanchard-Laville, C. (2008). Effets d'un cadre clinique groupal sur le travail du penser des participants. Approche psychanalytique. Dans M. Cifali et F. Giust-Desprairies (dir.), Formation clinique et travail de la pensée (p. 87-105). Bruxelles : Éditions De Boeck.

Blanchard-Laville, C. (2010). À l'écoute des enseignants. Violences dans le lien didactique. Revue de psychothérapie psychanalytique de groupe, 55, 147-162. doi : 10.3917/rppg.055.0147 
Blanchard-Laville, C. (2013a). Au risque d'enseigner. Pour une clinique du travail enseignant. Paris : Presses Universitaires de France.

Blanchard-Laville, C. (2013b). Du rapport au savoir des enseignants. Journal de la psychanalyse de l'enfant, 3(1), 123-154.

Blanchard-Laville, C., Chaussecourte, P., Hatchuel, F. et Pechberty, B. (2005). Recherches cliniques d'orientation psychanalytique dans le champ de l'éducation et de la formation. Revue française de pédagogie, 151(1), 111-162.

Blanchard-Laville, C. et Geffard, P. (2009). Processus inconscients et pratiques enseignantes. Paris : L'Harmattan.

BOEN (2003). $N^{\circ} 25$. Consulté sur http://www.education.gouv.fr/bo/2003/25/MENE0301199A.htm.

Bouveresse-Quilliot, R. et Quilliot, R. (1991). Les critiques de la psychanalyse. Paris : Presses Universitaires de France.

Canguilhem, G. (1994). Du singulier et de la singularité en épistémologie biologique. Dans Études d'histoire et de philosophie des sciences concernant les vivants et la vie (p. 211-225). Paris : Libraire Philosophique J. Vrin. (Texte original de 1962.)

Castarède, M.-F. (1983). L'entretien clinique à visée de recherche. Dans C. Chiland (dir.), L'entretien clinique (p. 118-145). Paris : Presses Universitaires de France.

Charrier, B. (2009). Approche clinique du travail en équipe pédagogique. Vers un rapport au savoir d'équipe? Analyses de réunions concernant trois équipes d'enseignant(e)s de Lettres modernes. (Thèse de doctorat, Paris Ouest Nanterre La Défense).

Chaussecourte, P. (2010a). Observer seul, penser à plusieurs : modalités d'accompagnement clinique d'un chercheur. Dans $M$. Cifali, M. Théberge et $M$. Bourassa (dir.), Cliniques actuelles de l'accompagnement (p. 139-157). Paris : L'Harmattan.

Chaussecourte, P. (2010b). Un dispositif pour conscientiser et élaborer son implication subjective lors d'observations directes. Actes du congrès de l'Actualité de la recherche en éducation et en formation, Université de Genève. Repéré sur le site du congrès international de I'AREF 2010 : https://plone.unige.ch/aref2010/symposiums-longs/coordinateurs-en-b/lanalyseclinique-des-pratiques-professionnelles-une-methode-pour-la-formation-et-larecherche-en-sciences-de-leducation/Un dispositif pour conscientiser.pdf/view

Chaussecourte, P. (2014). Le transfert didactique de Benoît : quelles évolutions ? Dans P. Chaussecourte (dir.), Enseigner à l'école primaire dix ans avec un professeur des écoles (p. 69-105). Paris : L'Harmattan.

Chaussecourte, P. (2017). Autour de la question du « contre-transfert du chercheur » dans les recherches cliniques d'orientation psychanalytique en sciences de l'éducation. Cliopsy, 17, 107-127

Cifali, M. (1999). Métier « impossible » ? Une boutade inépuisable. Le Portique, 4, 111.

Cifali, M. (2008). Une pensée affectée pour l'action professionnelle. Dans M. Cifali et F. Giust-Desprairies (dir.), Formation clinique et travail de la pensée (p. 129-147). Bruxelles : Éditions De Boeck Université.

Devereux, G. (2012). De l'angoisse à la méthode dans les sciences du comportement. Paris : Flammarion.

Engel, P. (1998). Réinventer la philosophie générale. Le Débat, 101(4), 157-164.

Freud, S. (1981). Le Moi et le Ça. Dans A. Bourguignon (dir.), Essais de psychanalyse (p. 219-275). Paris : Éditions Payot. (Texte original publié en 1923.)

Freud, S. (1985). Le créateur littéraire et la fantaisie. Dans L'inquiétante étrangeté et autres essais (p. 33-46). Paris : Éditions Gallimard. (Texte original publié en 1908.)

Freud, S. (1988). Le mot d'esprit et sa relation à l'inconscient. Paris : Éditions Gallimard. (Texte original publié en 1905.)

Freud, S. (2013). L'inconscient. Paris : Éditions Payot \& Rivages.

Gaillard, G. (2008). Se prêter à la déliaison. Narcissisme groupal et tolérance au féminin dans les institutions. Connexions, 90(2), 107-121. 
Gaillard, G. (2018). Tolérer l'effraction, travailler à inclure. Dans L.-M. Bossard (dir), Clinique d'orientation psychanalytique en éducation et formation. Nouvelles recherches (p. 103-121). Paris : L'Harmattan. (Texte original publié en 2011.)

Hegel, G. W. F. (1941). Phénoménologie de l'esprit. Paris : Éditions Montaigne. (Texte original publié en 1807.)

Kaës, R. (1975). Quatre études sur la fantasmatique de la formation et le désir de former. Dans R. Kaës (dir.), Fantasme et formation (p. 1-75). Paris : Dunod.

Kambouchner, D. (2007). L'éducation, question première. Revue de métaphysique et de morale, 56(4), 415-427. doi: 10.3917/rmm.074.0415

Mosconi, N. (1986). De l'application de la psychanalyse à l'éducation. Revue française de pédagogie, 75, 73-79.

Mosconi, N., Beillerot, J. et Blanchard-Laville, C. (2000). Formes et formations du rapport au savoir. Paris : L'Harmattan.

Pinto, L. (1983). L'école des philosophes. La dissertation de philosophie au baccalauréat. Actes de la recherche en sciences sociales, 47-48, 21-36.

Pinto, L. (2000). L'inconscient scolaire des philosophes. Actes de la recherche en sciences sociales, 135, 48-57.

Poucet, B. (1999). Enseigner la philosophie. Histoire d'une discipline scolaire. 18601990. Paris : CNRS Éditions.

Pujade-Renaud, C. (2005). Le corps de l'enseignant dans la classe. Paris : L'Harmattan.

Revault d'Allonnes, C. (dir.) (1989). La démarche clinique en sciences humaines. Documents, méthodes, problèmes. Paris : Dunod.

Rinaudo, J.-L. (2015). Être à l'école numérique aujourd'hui. Le sujet dans la cité, 6(2), 103-112.

Roman, J. (1988). La parole enseignante. Dans J. Message, J. Roman et E. Tassin (dir.), À quoi pensent les philosophes? (Vol. 102, p. 166-169). Paris : Autrement Revue.

Yelnik, C. (2005). L'entretien clinique de recherche en sciences de l'éducation. Recherche et formation, 50, 133-146.

Yelnik, C. (2007). Le contre-transfert comme outil de connaissance dans une recherche clinique en sciences de l'éducation. Actualité de la recherche en éducation et en formation, Strasbourg. Repéré sur le site du congrès international de I'AREF 2007 :

http://www.congresintaref.org/actes_pdf/AREF2007_Catherine_YELNIK_198.pdf

Dominique Renauld

CREF (EA 1589)

Université Paris Nanterre

Pour citer ce texte :

Renauld, D. (2019). Mise en scène de la parole magistrale dans un cours de philosophie. Cliopsy, 21, 89-104. 